\title{
The Effect of Thyroid Volume on Radioiodine Therapy Outcome in Hyperthyroid Cats
}

Veerle Volckaert, Eva Vandermeulen, André Dobbeleire, Luc Duchateau, Jimmy H Saunders, Kathelijne Peremans

Introduction: Radioiodine therapy in hyperthyroid cats is commonly used and has a high success rate ranging from $85 \%$ to $95 \%$. However given the drawbacks associated with a final hypothyroid or hyperthyroid outcome, a quest for causes of therapy failure is justified. As in human medicine, thyroid volume has been reported as a factor influencing radioiodine therapy outcome in hyperthyroid cats.

Purpose: The goal of this study was to relate total thyroid volume, calculated by a newly constructed formula for feline patients, to the outcome of radioiodine therapy. We hypothesized that larger volumes would result in a higher percentage of persistent hyperthyroidism and smaller volumes would increase the risk for hypothyroidism.

Materials \& Methods: For the calculation of total thyroid volume a new formula was introduced $\left(0.438^{*} \mathrm{~L}^{*} \mathrm{~W}^{2}\right)$. One hundred and sixty-seven hyperthyroid cats were included in search of a correlation between total thyroid volume and therapy outcome. Additionally the relationship between pretreatment total $\mathrm{T}_{4}$ and therapy outcome was investigated in 62 cats. Patients were categorized according the administered radioiodine dose and therapy outcome. The effect of volume and TT4 on the outcome was modeled by a logistic regression model with generalized logits, with the euthyroid cats as a reference category, and with dose as categorical, and volume and TT4 as continuous fixed effects.

Results: All patients had a follow-up of at least 6 months after the radioiodine therapy, $66.5 \%$ of them were euthyroid, $24 \%$ were hypothyroid and $9.5 \%$ were hyperthyroid. $31.7 \%$ of our patients were unilaterally affected, $56.9 \%$ were bilaterally affected and $11.4 \%$ were either unilaterally or bilaterally affected with additional ectopic thyroid tissue. A borderline significant increase $(P=0.0528)$ in the odds of hyperthyroidism relative to the odds of euthyroidism with increasing thyroid volume was found. Concerning the pretreatment total $\mathrm{T}_{4}$ concentration no significant relationships were detected.

Conclusion: The influence on therapy outcome is most likely multifactorial and although only a small effect was observed in this study, large thyroid volumes can increase the risk of a hyperthyroid outcome after radioiodine therapy. Surprisingly, in the two higher dose categories larger total thyroid volumes were also observed in final hypothyroid cats, compared to those with a euthyroid outcome, and the majority of them were bilaterally affected, possibly introducing another contributing factor on therapy outcome. No significant effect of pretreatment total $\mathrm{T}_{4}$ on outcome was observed.

Max 385 words in length (excluding title and author details). 